
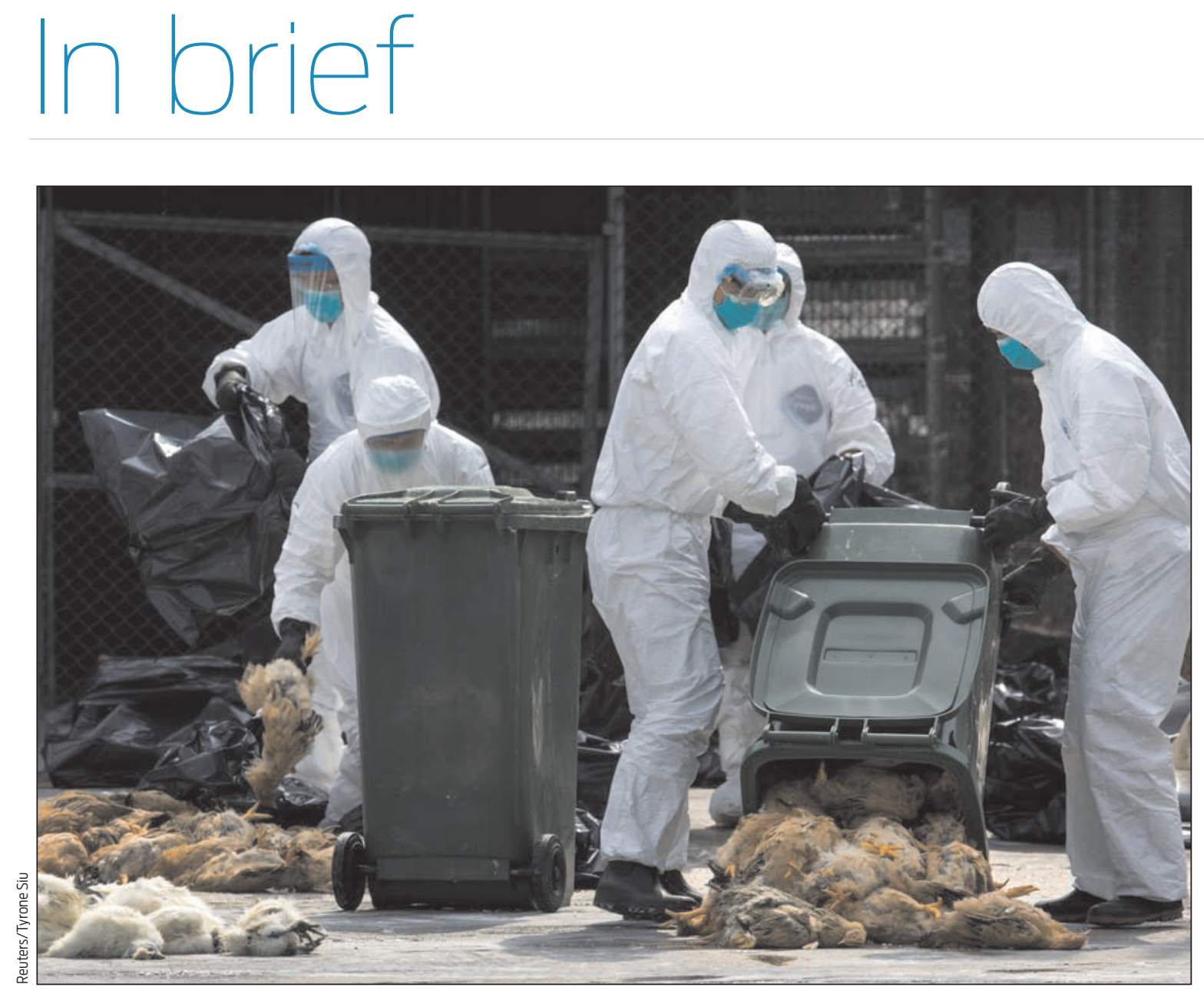

Health workers pack dead chickens into garbage bins at a wholesale poultry market in Hong Kong. Hong Kong began culling 20000 chickens and suspended imports of fresh poultry from mainland China for 21 days late in January after the discovery of the H7N9 bird flu virus in a batch of live chickens from the southern province of Guangdong.

\title{
From the CSIRO
}

\section{Creating a paradigm shift for diabetes dietary management}

Australian scientists are investigating how very low carbohydrate diets can be used to enhance diabetes treatment. The long-term clinical study involves the Commonwealth Scientific and Industrial Research Organisation (CSIRO), the University of Adelaide, the University of South Australia and Flinders University, and is supported by the National Health and Medical Research Council (NHMRC).

The obesity epidemic is leading an explosion in diabetes prevalence, with the World Health Organization reporting that 347 million people worldwide have diabetes (http://www. who.int/mediacentre/factsheets/fs312/ en/index.html), including 990000 people in Australia.

Lifestyle modification, diet and exercise are the cornerstones of diabetes management. Traditionally, the mainstream dietary approach has been to recommend following a high carbohydrate, low fat diet. However, this recommendation has been questioned following the new understanding that carbohydrate restriction and higher intakes of protein and unsaturated fats improve blood glucose control and cardiovascular disease risk.

The team has devised an eating pattern that markedly limits carbohydrates, increases protein and unsaturated fat, and is nutritious. This dietary approach has the potential to rapidly improve blood glucose control and lipid profile, therefore reducing overall cardiovascular risk in comparison with traditional dietary approaches. This is being tested in a large NHMRC-funded clinical trial with 120 participants with type

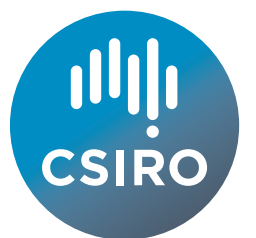

2 diabetes. Participants have been randomly allocated to consume either a traditional high carbohydrate, low glycaemic index, low fat diet or the very low carbohydrate, high unsaturated fat diet while participating in a structured exercise program.

The clinical study is still in progress, but preliminary findings are that independent of any change in weight, the blood glucose profiles improve substantially in the very low

Grant D Brinkworth Senior Research Scientist, CSIRO

Campbell H Thompson Professor,

University of Adelaide

CSIRO Animal, Food and Health Sciences

doi: 10.5694/mjal4.00097 carbohydrate group. The diet is well tolerated and the need for medication is also reduced to a greater extent. If improvements can be sustained, then this will have implications for dietary guidelines for the management of type 2 diabetes mellitus, with better outcomes and an overall reduction in health care costs. 


\section{MERS rears its ugly head}

Significant differences exist between the emergent coronavirus infection Middle East respiratory syndrome (MERS-CoV) and severe acute respiratory syndrome (SARS), including the presence of underlying comorbid conditions and the lack of an effective therapy for its severe extrapulmonary effects. A case series of

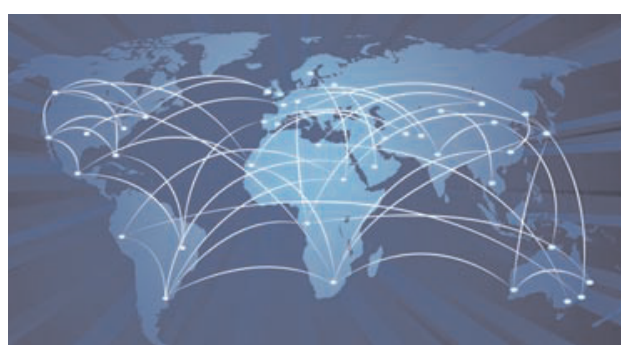
12 patients with confirmed or suspected MERS-CoV infection seen over 9 month in three intensive care units at two tertiary care hospitals in Saudi Arabia, published in Annals of Internal Medicine, found that all 12 patients had significant underlying comorbidities, including diabetes, renal disease and heart disease. All 12 presented with severe respiratory failure and hypoxia, and 11 had extrapulmonary symptoms including shock, acute renal injury, and thrombocytopenia. Five were alive at Day 90. An editorial said: "MERS-CoV does not yet have pandemic potential ... [however] we must [remember] the rapid evolution that occurred with SARS and that it emerged in a much more densely populated region. Given the right environment and a crowded part of the world, MERS-CoV might propagate more readily".

Ann Intern Med 2014; 28 January (online). doi: 10.7326/M13-2486; doi: 10.7326/M14-0096

\section{Pesticide traces linked with higher risk of Alzheimer}

Elevated serum levels of dichlorodiphenyldichloroethylene (DDE), a metabolite of the pesticide dichlorodiphenyltrichloroethane (DDT) are associated with an increased risk of late-onset Alzheimer disease (AD). Research from the US published in JAMA Neurology evaluated existing samples from $86 \mathrm{AD}$ patients and 79 controls and found that levels of DDE were 3.8-fold higher in the serum of those with AD compared with controls. The highest tertile of DDE levels was associated with an odds ratio of 4.18 for increased risk for AD. Additionally, patients carrying an APOE 4 allele in the highest tertile of DDE scored lower (-1.753 points) on the Mini-Mental State Examination compared with those carrying an APOE 3 allele. "Identifying people who have elevated levels of DDE and carry an APOE 4 allele may lead to early identification of some cases of $\mathrm{AD}^{\prime \prime}$, the authors concluded. JAMA Neurol 2014; 27 January (online). doi: 10.1001/jamaneurol.2013.6030

\section{Global atlas highlights palliative shortfalls}

Only one in 10 people globally who need palliative care receive it, says a report from the World Health Organization and the Worldwide Palliative Care Alliance. Australia's region, the West Pacific, has the largest fraction of adults needing palliative care, at $29 \%$ of the 20 million worldwide. The report said that 378 out of every 100000 Australian adults require access to palliative care services at the end of life, the second highest rate in the world. Dr Yvonne Luxford, CEO of Palliative Care Australia, said: "The West Pacific region has the highest percentage of adults (41.5\%) and children (14\%) in need of palliative care for a cancer

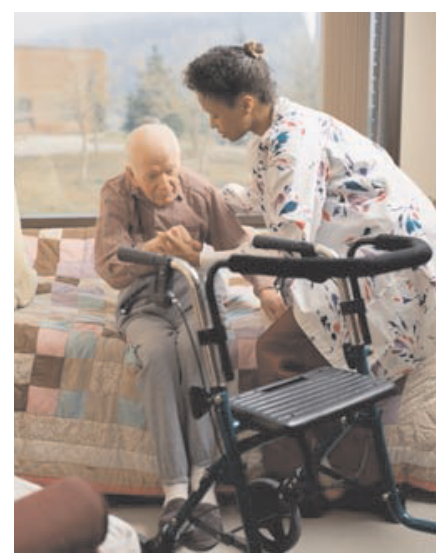
diagnosis". Australia has a disproportionately high

level of access for cancer patients and there was a need to boost support for those with a "variety of diseases, such as heart disease and dementia", she said. "The [report] gives us the data needed to position palliative care higher on national agendas."

WHO, WPCA 2014; The global atlas of palliative care at the end of life

\section{New Comments section in the MJA}

Readers are invited to email us a brief comment (no more than 100 words) on any current health-related matter, such as the state of our hospitals, junior doctors, Indigenous health, general practice, Medicare copayments, rural medicine, alcohol and our emergency departments, obesity or MJA themes. Note that comments about specific articles published in the MJA should be submitted as Letters to the Editor. Comments may be edited and will not be sent back to the author for approval. Publication is at the Editor's discretion; you will be notified if your comment is selected. Please include your full name, discipline and state of residence, and disclose any relevant information or affiliations that may affect interpretation of your comments.

Email your comments to: comments@mja.com.au

\section{MJA InSight poll}

Should workplace-based assessment be expanded for international medical graduates?

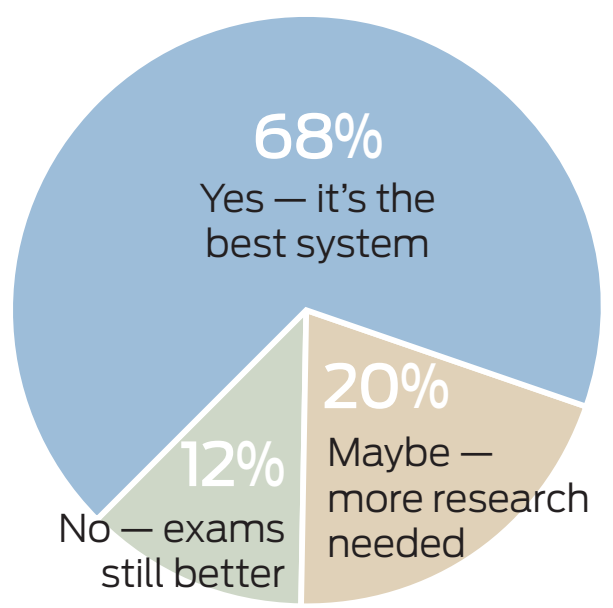

MJA InSight

Take part in next week's poll on www.mja.com.au/insight

Cate Swannell doi: 10.5694/mjal4.n0217 